\title{
Assessment of Correlations among Activities of Daily Living (ADL), Instrumental Activities of Daily Living (IADL) and Cumulative Illness Rating Index (CI) Scores in the Elderly Patients with Femur Fractures: A Prospective Study
}

\author{
Elsa Vitale $^{1^{*}} \quad$ Francesco Germini $^{2} \quad$ Michele Massaro $^{3} \quad$ Rosa Silvia Fortunato $^{4}$ \\ 1.PhD, MSN, BSN, ASL Bari, Italy \\ 2.MSN, BSN, RN, ASL Bari, Italy \\ 3.MSN, BSN, ASL Bat, Italy \\ 4.BSN, RN, ASL Bologna, Italy
}

\begin{abstract}
Background: The World Health Organization (WHO) has established that injury will be the second principal cause of the world disease onerous by the year 2020. Purpose: In this study comorbidity was evaluated with the Cumulative Illness Rating Index, (CI) and studied in relation to the functional status of the elderly participants, by considering: Activities of Daily Living (ADL), and Instrumental Activities of Daily Living (IADL), scores. Method: Ninety-five elderly patients with femur fracture were recruited for this study. A prospective investigation and evaluation of their comorbidity and their physical activity conditions was performed. Results: Correlations were evaluated between: ADL and CIRS values $(r=0.23)$; CIRS and IADL values $(r=0.24)$, and finally correlations between patients age and CIRS values $(\mathrm{r}=0.09)$. Conclusion: No significant correlations were assessed to standardize the different score levels.
\end{abstract}

Keywords: Activities of Daily Living (ADL), Cumulative Illness Rating Index (CI), Instrumental Activities of Daily Living (IADL), Elderly Patients.

DOI: $10.7176 / \mathrm{JHMN} / 63-05$

Publication date:June $30^{\text {th }} 2019$

\section{Introduction}

The ageing process is a biological certainty which has its own dynamic, largely beyond human control. In the developed countries, chronological time plays a primary role. The age of 60 or 65 is said to be the beginning of old age. In many parts of the developing world, chronological time has little or no importance in the meaning of old age. Other socially constructed meanings of age are more significant such as the roles assigned to older people; in some cases it is the loss of roles accompanying physical decline which is significant in defining old age. Thus, in contrast to the chronological milestones which mark life stages in the developed world, old age in many developing countries is seen to begin "at the point when active contribution is no longer possible".

Most developed world countries have accepted the chronological age of 65 years as a definition of "elderly" or "older person", but like many westernized concepts.

Comorbidity and functional capacity are important indicators of health in elderly; in fact their loss leads to a rise in mortality.

The World Health Organization (WHO) has established that injury will be the second principal cause of the world disease onerous by the year 2020. A limitation in the current process of deriving population assessed of the trouble attributable to injury is the failure to consider pre-existing comorbidity. If damaged people diverge from the general population in terms of pre-existing comorbidity, then observed outcomes in injured samples that are attributed to injury may in part due to pre-existing comorbidities rather than to the injury in question.

In this study comorbidity was evaluated with the Cumulative Illness Rating Index (6), (CI). This scale is designed to measure the chronic medical illness burden in the elderly people. This is a 13 - category scale measuring elderly persons' cardiovascular - respiratory system, gastrointestinal system, genitourinary system, muscoloskeletal - integumentary system, neuropsychiatric system and general system. For each of the 13 organ system, the illness burden is rated on 5 point scale, as following, none, mild, moderate, severe, extremely severe. Total score calculated by summing the score on individual category.

Comorbidity conditions were studied in relation to the functional status of the elderly participants, by considering: Activities of Daily Living (ADL), and Instrumental Activities of Daily Living (IADL), scores.

ADL describe activities essential for self - care like bathing, dressing and feeding. The ADL scale represents the most basic activities involved in every independent function. A summary score ranges from 0 to 6 .

The Lawton Instrumental Activities of Daily Living Scale (IADL) is an appropriate instrument to assess independent living skills. These skills are considered more complex than the basic activities of daily living as measured by the Katz Index of ADLs. The instrument is most useful for identifying how a person is functioning at the present time and for identifying improvement or deterioration over time. There are 8 domains of function 
measured with the Lawton IADL scale. Historically, women were scored on all 8 areas of function; men were not scored in the domains of food preparation, housekeeping, laundering. However, current recommendations are to assess all domains for both genders. Persons are scored according to their highest level of functioning in that category. A summary score ranges from 0 to 8.

The aim of the present study is to evaluate the relation existed between the CI index and the functional status in the elderly, by considering also age of participants as another dependent variable.

\section{Methods}

Ninety-five elderly patients with femur fracture were recruited for this study. A prospective investigation and evaluation of their comorbidity and their physical activity conditions was performed. Participants were recruited from the admissions of femur fracture in the Orthopedic Ward at the General Hospital in Bari, in the Southern of Italy. Patients with a history of past hip fracture or bilateral hip fracture were excluded. Also patients with malignant bone disease were excluded.

Professionals in charge of doing and collecting data were trained to unify criteria. Participants were assessed in the General Hospital of Bari. Before the data collection, all participants were informed about the study and confirmed their oral corresponding informed consent. Functional status was evaluated by considering ADL, and IADL scores; while co morbidity assessment was performed thanks to CIRS index.

\section{Results}

The average age of the studied population was $78.65 \pm 18.97$ years for men and $84.17 \pm 6.97$ for women.

Correlations were evaluated between: ADL and CIRS values ( $r=0.23)$; CIRS and IADL values $(r=0.24)$, and finally correlations between patients age and CIRS values $(\mathrm{r}=0.09)$.

There were not any strong correlations between activity daily scores and co morbidity index.

So, no correlations there were between these scores to standardize the different score levels. Moreover, ADL and IADL were also not correlated, if we consider different gender.

\section{Discussion}

Taking into account the data from the European Community Household Panel Survey (ECHP) referring to people over 65 in the European Community, it is observed that the European Union is ageing as a result of two developments: firstly, the number of people aged 65 years and over is increasing and, secondly, the number of children (age group 0 - 14 years) is decreasing. Although in 2001 rural areas had on average an older population than intermediate or urban areas, from 2001 to 2006 the share of the old age group grew faster in urban areas. Also, in Italy, there was a clear geographical divide but this time on a north south basis, the population of the north increasing while that of the south decreased. Also elderly increases their dependence to carry out some activities of daily living which corresponds to $35 \%$ of the population older than 65 .

Literature showed that increasing age is generally considered a major determinant of health status on its own, with a consistently reported age - related decline in functional status and an increase in disability rates. Additionally, in elderly patients functional status constitutes an important indicator of the overall health condition, reflecting the degree of an individual's dependence and his ability to use health care services. Moreover, functional status represents a good predictor of mortality.

In the Waldman's study (1992) the Cumulative Illness Rating Scale was examined in a logistic regression with activities of daily living and it was demonstrated that the Cumulative Illness Rating Scale was a significant predictor of death, yet it did not improve that prediction over information contained in measures of activities of daily living.

In the Extermann's study it was highlighted that co morbidities needed to be assessed independently from functional status.

In this study possible correlations between Cumulative Illness Rating Scale and activities daily living indexes was assessed, but results demonstrated that the association of more illnesses could not be significant associated with functional disability in the elderly. Moreover, women had less dependence ratios than men. Nevertheless, it is necessary to go deeper in this aspect before reaching more accurate conclusions.

Regarding the functional status and based on these results, all the participants, both men and women, had partially dependence; however women seemed to be more functional active than men.

$20 \%$ of the elderly participants were categorized as independent to carry out all ADL assessed; while $14.77 \%$ were independent for IADL. Therefore, the highest level of dependence corresponds to the ADL since those are activities requiring greater physical integrity and imply the necessity of higher cognitive integrity that the ADL.

When considering gender and IADL assessed, men present a higher level of dependence than women, which means that in general, they are more dependent than women in the assessed area, although these differences are not strong enough to be considered relevant. However, considering each of the different activities of the index in a separate way and taking account gender, it shows that there are differences among the categories of each item. 
Finally, advanced age both in male and female subjects, ADL and IADL scores were not associated significantly with co morbidity index, too. So, more Cumulative Illness Rating Score will be observed in the elderly, but difficulties in their functional status exist will not be predicted.

\section{Acknowledgement}

The authors gratefully acknowledge all studies' authors included in this review for their commitment in this field.

\section{References}

Gorman, M. Development and the rights of older people. The ageing and development report: poverty, independence and the world's older people. London: Earthscan Publications Ltd,1999.

Togunu-Bickersteth, F. Chronological definitions and expectations of old age among young adults in Nigeria. Journal of Aging Studies, 1987; 1 (2), 113-124.

Stuck, AE, et al. A randomized trial of in-home visits for disability prevention in community-dwelling older people at low and high risk for nursing home admission. Archives of internal medicine, 2000; 160, 977-986.

Guralnik, JM, et al. Preventing disability through community-based health coaching. Journal of the American Geriatrics Society, 2003; 51, 265-269.

Mehr, DR, Tatum, PE. Primary prevention of diseases of old age. Clinics in geriatric medicine, 2002; 18 , 407430.

Linn, BS, Linn, MW, Gurel, L. Cumulative illness rating scale. J Amer Ger Soc, 1968; 16, 622-626.

Katz, S., Ford, A.B., Moskowitz, R.W., et al. Studies of illness in the aged. The Index of ADL: a standardized measure of biological and psychosocial function. JAMA, 1963; 185, 914- 919.

Lawton, M.P., Brody, E.M. Assessment of older people: Self-maintaining and instrumental activities of daily living. The Gerontologist, 1969; 9, 179-186.

Lawton, M.P., Moss M., Fulcomer M., Kleban, M. H. Multi-level assessment instrument manual for full-length MAI. North Wales, PA: Polisher Research Institute, Madlyn and Leonard Abramson Center for Jewish Life, 2003.

European Commission. Eurostat yearbook; 2010. Available at: http://epp.eurostat.ec.europa.eu.

Repetto, L., et al. Comprensive Geriatric Assessment Adds Information to Eastern Cooperative Oncology Group Performance Status in Elderly Cancer Patients: An Italian Group for Geriatric Oncology Study. Journal of Clinical Oncology, 2002; 20, 494-502.

Waldman, E., Potter, JF. A prospective evaluation of the cumulative illness rating scale. Aging, 1992; 4, 171-178.

Extermann, M., et al. Comorbidity and functional status are independent in older cancer patients. American Society of Clinical Oncology, 1998.

Ali H., Ibrahem S.Z., Al Mudaf B., Al Fadalah1 T., Jamal D., El-Jardali F. Baseline assessment of patient safety culture in public hospitals in Kuwait. BMC Health Services Research. 2018; 18:158.

Alquwez, N., et al. Nurses' Perceptions of Patient Safety Culture in Three Hospitals in Saudi Arabia. Journal of Nursing Scholarship, 2018, 50:4, 1-10.

da Costa D. B., Ramos D, Gabrie C.S., Bernardes A. Patient safety culture: evaluation by nursing professionals. Texto Contexto Enferm. 2018; 27(3):e2670016.

Farokhzadian J., Nayeri N.D., Borhani F. The long way ahead to achieve an effective patient safety culture: challenges perceived by nurses. BMC Health Services Research. 2018; 18 : 654.

Gallen, A. et al. How do nurses and midwives perceive their preparedness for quality improvement and patient safety in practice? A cross sectional national study in Ireland. Nurse Educ Today, 2019 May;76:125-130

Health and Safety Commission Advisory Committee on the Safety of Nuclear Installations. Organizing for safety: Third report of the ACSNI study group on human factors. Sudbury, UK: HSE Books. 1993.

Hellings J., Schrooten W., Klazinga N., Vleugels A. Challenging patient safety culture: survey results. Int. J. Health Care Qual. Assur 2007; 20 (7): 620-632.

Huang C.H., WU H.H., Chou C.Y., Dai H., Lee C.Y. The perceptions of physicians and nurses regarding the establishments of patient safety in a regional teaching hospital in Taiwan. Iran J Public Health. $2018 ; 47$ (6): $852-860$

Hurtado D.A., Heinonen G.A., Dumet L.M., Greenspan S.A. Early career nurses with fewer supportive peers for safe patient handling are likely to quit. International Council of Nurses. 2018.

Institute of Medicine.To Err is human: Building a safer health system. Washington, DC, 1999: National Academies Press. https://doi.org/10.17226/9728. accessed August 2018.

Islam, F.; Rashid, M. Evaluating Nurses' Perception of Patient Safety Design Features in Intensive Care Units. Crit Care Nurs Q. 2018, Jan/Mar;41(1):10-28.

Kanaskie M.L., Snyder C., et al. Nurses and nursing assistants decision-making regarding use of safe patient handling and mobility technology: A qualitative study. Applied Nursing Research. 2018; 39: 141-147.

Kohn L.T., Corrigan J.M., Donaldson M.S. To Err Is Human: Building a Safer Health System. National Academy 
Press, Washington, 1999.

Kohn L.T., Corrigan J.M., Donaldson M.S. To err is human: Building a safer health system. Washington, DC: National Academy Press. 2000.

McCarthy D., Blumenthal D. Stories from the sharp end: case studies in safety improvement. Milbank Q. 2006; 84 (1): 165-200.

Melnyk B.M., Gallagher-Ford L., Zellefrow C., Tucker S., Bindu T. The First U.S. Study on Nurses' EvidenceBased Practice Competencies Indicates Major Deficits That Threaten Healthcare Quality, Safety, and Patient Outcomes. Worldviews on Evidence-Based Nursing. 2018; 15 (1): 16-25.

Nieva V.F., Sorra J. Safety culture assessment: a tool for improving patient safety in healthcare organizations. Qual. Saf. Health Care. 2003; 12 (Suppl. 2): ii17-ii23.

Singer S., Lin S., Falwell A., Gaba D., Baker L. Relationship of safety climate and safety performance in hospitals. Health Serv. Res. 2009; 44: 399-421.

Titlestad I., Haugstvedt A., Igland J., Graue1 M. Patient safety culture in nursing homes, a cross-sectional study among nurses and nursing aides caring for residents with diabetes. BMC Nursing. 2018; 17:36. 